\title{
Cherry-Picking Customers by Party Size in Restaurants
}

\author{
Gary M. Thompson \\ School of Hotel Administration \\ Cornell University \\ Ithaca, New York, NY \\ Email: gmt1@cornell.edu
}

Keywords: revenue management, customer service, restaurants

Author's Note: Gary M. Thompson is a Professor of Operations Management in the School of Hotel Administration at Cornell University, where he teaches graduate and undergraduate courses in Service Operations Management. He holds a B.Sc. with First Class Honors (University of New Brunswick), an MBA (University of Western Ontario), and a PhD in Operations Management (The Florida State University). His current research focuses on restaurant revenue management, food and beverage forecasting in lodging operations, and wine cellars. His research has appeared in the Cornell Hospitality Quarterly, Decision Sciences, the Journal of Operations Management, the Journal of Service Research, Management Science and other journals.

Acknowledgment: The author would like to thank the owner of Casual Restaurant for participating in the student project that was the genesis for this research.

Declaration of Conflicting Interests: The author(s) declared no potential conflicts of interest with respect to the research, authorship, and/or publication of this article.

Funding: The author(s) received no financial support for the research, authorship, and/or publication of this article. 


\title{
Cherry-Picking Customers by Party Size in Restaurants
}

\author{
Abstract \\ This paper examines the phenomenon of cherry-picking customers—serving the high value customers and \\ denying service to low value customers. These actions are fundamental to revenue management, but have \\ received essentially no attention in the context of restaurants. Two common characteristics of restaurants \\ make them a logical environment in which to implement cherry-picking—compared to smaller parties, \\ larger parties typically take longer to dine and spend less per person. A short example provides \\ motivation and the author reports on an international survey of restaurant managers, which indicates that \\ cherry-picking actions are not uncommon. The author also reports on a simulation study to explore the \\ factors affecting the level of excess demand necessary to justify cherry-picking. Important factors are \\ customers' willingness to wait for tables, the length of the peak-demand window, and the proportionality \\ of space required by different size tables. The author also evaluates two heuristics for predicting the \\ viability of cherry-picking. The author believes that the most important finding for managers is that the \\ viability of cherry-picking is very dependent on restaurant size, being generally impractical for larger \\ restaurants. From an academic perspective, since this is under-investigated topic, the author identifies \\ nine research extensions of this work, ranging from empirical to analytical investigations.
}




\section{Introduction}

The issue of cherry-picking — picking the low-hanging, high value items—-has been applied in marketing contexts from the perspective of customers-examining how customers might switch between retailers to find the lowest priced items, for example. Our perspective in this paper is that of the firm, and we look at cherry-picking of customers. Cherry-picking of customers is consistent with the revenue management goal of selling the right capacity to the right customer at the right time and right price (Smith, Leimkuhler, and Darrow 1992). However, we can find no literature examining cherry-picking in the context we consider-restaurants—which is somewhat surprising given the size of the industry. 2010 sales in the U.S restaurant industry are forecast to be $\$ 388.5$ billion (Anonymous 2010).

Two characteristics of restaurants make them logical candidates for cherry-picking customers based on party size. First, compared to smaller parties, large parties typically spend less per person. Second, larger parties typically take longer to dine. Thus, large parties are the logical candidates for not being served. In other words, many restaurants are in a situation to consider cherry-picking the smaller, more valuable parties.

The motivation for the current study came from a master's level course in restaurant revenue management, taught by the author, which used an independent restaurant in Ithaca, NY as a case study. The restaurant in question, which we shall refer to as Casual Restaurant, is on the small side, with only 42 seats. It does not take reservations, but relies totally on walk-in business. It has 6 two-seat tables, 6 fourseat tables, and 6 seats at the bar. Its average party size is approximately 2.55 people. While the restaurant does not explicitly state that they will not take larger parties, it purposefully discourages larger parties from dining by quoting to such parties arbitrarily long wait times for tables. Though the large parties are less valuable on a space-time metric, turning away the larger parties would only make sense if there is sufficient demand of smaller parties to replace the lost business. Casual Restaurant is one of the more popular restaurants in the local area, so the restaurant owner's actions—of cherry-picking only smaller parties—may make good business sense. 
We have three main goals for this paper. First, we report on an international survey we conducted to see how common cherry-picking practices are in restaurants. Second, we conduct a simulation experiment of a number of restaurant contents, with the purpose of identifying when cherry-picking is practical. Third, we develop and test two heuristics that managers might use for predicting whether their restaurants have sufficient excess demand to justify party-size-based cherry-picking. We find that restaurant size, relative space requirements across table sizes, differences in the per-person average checks across party sizes, differences in dining durations across party sizes, customers’ willingness to wait for a table, and the length of the peak demand period all affect the viability of cherry-picking based on party sizes. We also find that the heuristic we developed for predicting cherry-picking viability that uses information on customers' willingness to wait for service yields much more accurate predictions than a simpler heuristic that ignores this information.

Our focus here is on static cherry-picking — a priori decisions about which customers will be served and which will not. Though cherry-picking may also be applied on a dynamic basis, as customers arrive, we leave that investigation to future research.

The remainder of the paper has the following structure. We first review relevant literature and then describe the international survey we conducted. We introduce two heuristics for estimating the level of demand intensity that is necessary to justify cherry-picking. We describe and present the results of the simulation experiment we conducted. We discuss our findings and close with our conclusions.

\section{Literature Review}

The literature on applying revenue management concepts to restaurants has been growing since the term “'Restaurant Revenue Management'” (RRM) was first coined by Kimes et al. (1998). Much of the RRM literature has focused on capacity management issues, such as identifying the optimal table mix for restaurants (Kimes and Thompson 2004; Thompson 2002) or on reservations policies (Bertsimas and Shioda 2003, Thompson and Kwortnik 2008). Demand- or customer-focused RRM-related research 
includes that of Kelly, Kiefer, and Burdett (1994), who examined how menu pricing can affect item demand; Kimes, Wirtz, and Noone (2002), who looked at customers' expected meal durations in North

America, Europe, and Asia; Kimes and Robson (2004), who studied how customers’ spending and meal duration is affected by the characteristics of the tables to which they are assigned; McGuire and Kimes (2006), who examined how four approaches for managing restaurant waitlists are viewed by customers; and Robson and Kimes (2009), who studied customer reactions to characteristics of restaurant tables. Bertsimas and Shioda (2003) developed mathematical models for deciding which customers to accept in restaurants having walk-in customers; their focus was not, however, on segmenting the customers by party size.

Kimes et al. (1998) introduced a time-and-capacity based performance measure, which they called RevPASH, or Revenue Per Available Seat Hour, to correspond to the commonly used lodging metric RevPAR (Revenue per Available Room). This metric offers a means of comparing different party sizes. Several studies have reported that dining duration increases as party size increases (Bell and Pliner 2003; Kimes and Robson 2004; Kimes and Thompson 2004). It has also been observed that larger parties tend to spend less per person than smaller parties (Kimes and Robson 2004; Kimes and Thompson 2004). These two characteristics result in lower RevPASH for larger parties than for small parties, making the smaller parties candidates for being cherry-picked.

Kimes (2004) argued for there being two strategic levers for applying revenue management in restaurants: price and duration. Price-related decisions include such things as offering early-bird discounts and differentiating prices across meal periods or by location within the restaurant (such as premium pricing for sitting at a chef's table). Duration-based decisions can involve redefining duration, reducing arrival uncertainty, reducing duration uncertainty, and shortening the time between meals (Kimes 2004). Missing from this two-level framework is the "right customer" component of traditional revenue management: deciding which customers should be served, and which, if any, should be turned away. We are not aware of any research in the context of restaurants that addresses the serve/turn-away decision. 
The topic, however, falls within the “customer segmentation”' topic, one of the seven topics on managing customers for value for which Kumar, Lemon, and Parasuraman (2006) suggested additional research. It also falls, we believe, within the "measuring and optimizing the value of service”' topic, one of the 10 service-related topics for which a recent article in this journal called for research (Ostrom et al. 2010). In the next section, we describe the international survey we conducted to examine the phenomenon.

\section{Restaurant Survey}

The survey we conducted was sent out to 2,100 people who had downloaded at least one of the author's restaurant-related reports or tools available from a U.S.-based, hospitality-oriented website. A reminder was sent via e-mail between 10 and 14 days after the initial survey. In total, 481 people responded to the survey, for a response rate of $22.9 \%$. Of the responders, 207 were not directly working in restaurants or food and beverage outlets in lodging properties, leaving 273 usable responses, with the geographical distribution reported in Table 1.

Of the qualified respondents, 37.6\% represented chain restaurants, while the remainder were independents. Standalone restaurants represented $16.4 \%$ of the respondents, with $72.5 \%$ of the respondents located in a lodging property, with the remainder (11.1\%) being in other locations such as strip malls. The restaurants ranged in size from 26 to 2,000 seats, with a mean of 211 seats and a median of 150 seats. A histogram of the restaurant sizes is illustrated in Figure 1.

We asked the question " Do you discourage or restrict certain size parties from dining in your restaurant/F\&B outlet, such as limiting party sizes to 6 or smaller?’' Of the 203 people who answered this question, 35 (or 17.2\%) responded in the affirmative. The actions taken for larger parties include:

- $\quad$ Turning the parties away;

- $\quad$ Restricting the times during which the parties will be accepted;

- $\quad$ Diverting the business to another part of the operation (such as banquets);

- $\quad$ Using restricted menus; 
- $\quad$ Switching to buffet service;

- $\quad$ Requiring deposits;

- $\quad$ Requiring minimum drink orders; and

- $\quad$ Allowing the purchase of the entire restaurant for an evening.

We also asked a question probing how wait times are quoted, "For walk-in parties, please identify how you prefer to quote estimated waiting times, for each party size.’' Figure 2 displays the results from this question, by restaurant size (the respondent restaurants for this question had a median of 140 seats). As party size increases from two to five people, the percentage of respondents who prefer to underestimate the wait decreases slightly, staying relatively stable for parties of five or more people. The percentage of respondents who prefer to accurately estimate the wait decreases sharply for parties over four people. The percentage of respondents who prefer to overestimate the wait increases as the party size increases from four to five people, and again as the party size increases from five to six people. For parties of one to six, the dominant preference is for accurately estimating the wait time; while for parties of seven and more, the dominant preference is for overestimating the wait. It is apparent from Figure 2 that, compared to larger restaurants, smaller restaurants more frequently overestimate the wait time for larger parties.

Figure 3 reports the same results as in Figure 2, but does so for the most common geographical locations in the response set. The most obvious geographical effect in Figure 3 is that respondent restaurants in the United States and Canada more frequently attempt to quote accurate waiting times across all party sizes, followed by respondent restaurants in Europe, with the lowest frequency from respondent restaurants in Asia. Respondent restaurants in the United States and Canada also had the lowest frequency of underestimating wait times, for all party sizes bigger than two people.

The results displayed in Figures 2 and 3 show that the phenomenon we observed in Casual Restaurant—quoting longer-than-expected waits for larger parties-is actually quite common. While we did not include a question in the survey about the motives that restaurant managers have for the wait times they quote, we believe that there are two possible motives. First, larger parties may inherently have more 
variation in wait times, so quoting longer waits would be a way of maintaining a service level, such as 95\% of parties being served within the stated wait. Second, it may be that the managers prefer to discourage larger parties, as with Casual Restaurant. Even if the former is the main motivation, a byproduct might well be that a smaller percentage of the larger parties are willing to wait for a table. As such, regardless of the main underlying motive, the wait time quoting preferences would suggest that the phenomenon we observed in Casual Restaurant occurs quite commonly. The next section describes the specific cherry-picking context we consider.

\section{Cherry-Picking Context}

As mentioned in the introduction, if a business purposefully chooses not to serve certain types of customers, and it wishes to be at least as profitable as it was when all customers were being served, there must be sufficient other customers available to offset the business being turned away. Alternatively, if a business excludes customers that simply are not profitable, then no additional customers need be served. However, in a restaurant setting, even the large parties are profitable, but perhaps not to the same degree as smaller parties. So, we focus on the level of excess demand that is necessary for cherry-picking to be more profitable than attempting to serve all customers. We define "full demand" as the level of customer demand that, in theory, is just within the capacity of the restaurant to serve. 'Excess demand," then, represents demand above the level of "full demand."

We are purposely going to keep the context simple: we will assume that each restaurant serves parties of one through 10 people, using tables with 2, 4, 6, 8, and 10 seats. We will assume that the restaurants exhibit the common phenomena of larger parties spending less per person and taking longer to dine, since these conditions are also necessary for size-based cherry-picking to occur. We are specifically going to focus on identifying the demand level that is necessary for the restaurant to stop serving parties of 9 and 10; that is, cherry-picking the smaller parties. We selected the parties of 9 and 10 as those to be dropped because parties of 9 had the highest instance of overestimating the waiting time (in Figures 2 and 3) and so it is those size parties that are the most likely to be dropped, either purposefully by the 
restaurant or by customers' self-selection. The next section develops two decision heuristics to predict the conditions under which it makes sense to turn away the largest parties.

One might question whether excluding parties of 9 and 10, by not providing a table large enough for them to be seated, is, in fact, an example of cherry-picking. We argue that it is. Most restaurants can, if they choose to do so, combine tables to seat larger parties. Casual Restaurant, for example, has no table bigger than four seats, but commonly combines tables to seat parties of five and six (but much less frequently combines tables to seat parties of seven or more). Consider an extreme case of a very large party, say 20 people. Few people would expect a restaurant to have a single table capable of seating such a large party, but people do expect that tables can be combined, as necessary, to seat their group. Cherrypicking exists if a choice is made to turn away certain customers to focus on others. As we noted earlier, for cherry-picking to be viable, the restaurant must have excess demand.

\section{Cherry-Picking Viability Heuristics}

In this section, we will describe two heuristics we developed to predict the viability of cherrypicking. The purpose of the heuristics is to predict the level of excess demand that is necessary to justify cherry-picking. If the actual level of excess demand exceeded the indicated level, then cherry-picking would be viable according to that heuristic.

DmdHeur1. The first heuristic is based on identifying the best substitute for a 10-seat table, based on expected values of the substitutes, and then determining how much demand would have to increase to replace the revenue lost when the 10 -seat tables are eliminated. Since this heuristic performed very poorly compared to the second heuristic we tested, we do not provide detailed information on it, but those details are available from the author.

DmdHeur2. The second heuristic incorporates information on customers' willingness to wait for a table through a minute-by-minute static estimation of parties arriving, parties waiting, and parties seated. The heuristic iteratively evaluates demand increases in increments of $1 \%$ of full demand. It stops when 
the revenue lost when all parties are served exceeds the revenue lost when cherry-picking is in effect, or when the demand has been inflated by 500\%, whichever comes first. The specific steps in this heuristic are described in the appendix. Our expectation is that the second heuristic will perform better than the first, simply because it offers a means of considering customers' willingness to wait. In the next section, we describe the simulation experiment we conducted to explore the effectiveness of these decision heuristics.

\section{Simulation Experiment}

The purpose of the simulation experiment was to examine a broad range of simulated restaurant environments, to identify the level of excess demand necessary to economically justify party-size-based cherry-picking. We conducted the experiment using a special-purpose simulation model written in Microsoft1 Visual Basic, which allowed us to control the disparate components of the experiment. The process followed for each of the 384 simulated environments (described below) was to set the full demand intensity such that the restaurant would be at, but not over, full capacity. Full demand intensity, in parties per hour, is given by:

FDI (space in restaurant)*(60 minutes)

$=\overline{\sum_{p \in \text { arty size }}\left[\left(\text { probability }_{p}\right) *(\text { dining duration for } p)(\text { space required by the table that best fits } p)\right]}$

By setting the initial demand level at full demand intensity, that is, the point where the restaurant would be at, but not over full capacity means that if a restaurant is going to turn away large parties, it must have other business on which it can rely. Next, demand (the overall party arrival rate) was repetitively inflated by $50 \%, 100 \%, 150 \%$, and $200 \%$, stopping as described below. The best table mix at a particular demand level was identified using a simulated annealing-based heuristic (SAH), much like that reported by Kimes and Thompson (2005). Those authors reported that their simulated annealing heuristic yielded tables mixes with revenue within $0.2 \%$ of that yielded by the optimal table mix found using an exhaustive 
search. Given the size of our experiment, and the range of table sizes we used, an exhaustive search was impractical.

Our SAH began by creating and storing information on 100 days of party information; that common information was used to evaluate each of the different table mixes examined. SAH then constructed an initial table mix using the Naïve Table Mix calculations (Thompson 2002) and then evaluated it and 99 additional table mixes (i.e., 100 mixes in total). Each of the alternate table mixes was obtained by making perturbations to an earlier identified mix. SAH maintained a list of previously evaluated mixes, to avoid wasting effort by resimulating a previously simulated mix. The best-performing of the 100 tables mixes evaluated was the one selected for comparison purposes.

At each level of demand, the revenue yielded by the best table mix using all table sizes (Value210) was compared to the revenue yielded by the best table mix using only tables of 2, 4, 6, and 8 seats (Value2-8). If Value2-8 exceeded Value2-10, then the simulation was run again at a demand level reduced by $25 \%$ of the full demand. In most instances, this allowed us to identify both upper and lower bounds on the demand level increase necessary to justify cherry-picking. However, in those instances where Value2-10 still exceeded Value2-8 at the 200\% increase in demand, we have only a lower bound on the necessary demand increase to justify cherry-picking. While ideally we would have tighter bounds on the level of demand necessary to justify cherry-picking, it is a very computationally intensive process to identify the exact demand level at which cherry-picking becomes viable.

Below we describe the design, identify the assumptions, and present the results of the simulation experiment.

Design of the simulation experiment. The full-factorial experiment had eight factors, as identified in Table 2. We describe each factor below. There were two levels for restaurant size: capacities for seating approximately 30 and 60 parties simultaneously (i.e., having about 30 and 60 tables). We used the number of tables, rather than a specified space area, since one of the factors described below varies the 
tables' space requirements. We used 30 tables because this is sufficient to ensure that the ideal table mix would contain at least one 10-seat table. Seating 60 parties simultaneously roughly corresponds to a restaurant size falling within the range defined by the median and mean number of seats in the restaurants from our survey. While it would have been desirable to include even larger restaurants in the study, simulating the larger restaurants becomes computationally prohibitive. In general, the flexibility in serving parties increases with larger restaurants, which leads us to expect that:

Hypothesis 1: Cherry-picking will be viable at lower levels of excess demand with the smaller restaurants.

Mean party size had two levels: 2.5 and 3.0 people. As we reported earlier, Casual Restaurant has a mean party size of 2.55 people. We are aware of only one instance in the literature of a mean party size being reported-approximately 2.6 customers per party (Kimes and Robson 2004). Both these values correspond well to the smaller mean party size we used. For completeness, we included the larger party size. Table 3 displays the probabilities of the different party sizes, for the two levels of this factor. Because smaller parties have a higher customer value per space-time unit, we expect that:

Hypothesis 2: Cherry-picking will be viable at lower levels of excess demand with the smaller party size

To capture the phenomenon that larger parties tend to spend less per person than do smaller parties (Kimes and Robson 2004; Kimes and Thompson 2004), we used two levels of variation in the average per-person check across party sizes. For both levels, the average spend was $\$ 10$ for parties of one, declining linearly to spends of $\$ 9$ and $\$ 8$ per person for parties of 10 . Since a requirement of party-sizebased cherry-picking is having smaller parties having a higher value per space-time unit, we expect that:

Hypothesis 3: Cherry-picking will be viable at lower levels of excess demand with greater differences in the spend per person. 
We believed that customers' willingness to wait for a table would have a notable effect on the practicality of cherry-picking. Having customers who are willing to wait longer for a table in effect gives the restaurant additional capacity, since the customers are acting as a demand inventory, which would result in less of the higher-value business being lost even if the lower-value customers are served. As such, we expect that:

Hypothesis 4: Cherry-picking will be viable at lower levels of excess demand when customers are less willing to wait for tables.

We are aware of no published studies on customers' willingness to wait for restaurant tables other than anecdotal evidence that suggests that larger parties are willing to wait longer for a table than are smaller parties (Field, McKnew, and Kiessler 1997), so we selected levels and factor levels that we believed would be representative of wide variety of restaurant contexts. We had three levels for customers’ willingness to wait: waiting tolerances of $105-120$, 45-60, and 15-30 minutes for parties of 1-10. The differences in wait tolerance between party sizes was the same, and linear, in all the levels. This assumption means, for example, that if a party of two people is willing to wait 45 minutes for a table on average and a party of four people is willing to wait an average of 50 minutes, then a party of six would be willing to wait an average of 55 minutes.

There were two levels of duration variation across party sizes, both reflecting the phenomenon of increasing dining duration with increasing party size that has been observed in several studies (Bell and Pliner 2003; Kimes and Robson 2004; Kimes and Thompson 2004). Again, this characteristic is one of the two that set the stage for party-size-based cherry-picking in restaurants. The two levels of this factor used a 1.5:1 and a 2.0:1 ratio of dining times for parties of 10 relative to the dining time for parties of one. These values fall within the range of this ratio observed in the literature, from 1.14 (Kimes and Thompson 2004) to 3.18 (Bell and Pliner 2003). With both factor levels, we imposed a linear relationship between mean dining duration and party size. Since greater differences in dining durations across party sizes also increase the discrepancy across party sizes of the value per space-time unit, we expect that: 
Hypothesis 5: Cherry-picking will be viable at lower levels of excess demand with greater variation in dining duration across party sizes.

Peak demand period length refers to the length of time that the customer arrival rate stays at its highest level. We used two levels for this factor: 2 and 4 hours, to reflect a broad range of restaurant settings. Customers who arrive toward the end of the peak period may well be served before their wait tolerance is exceeded, because as demand tails off the restaurant has the opportunity to start reducing the queue of waiting customers. With a longer peak, the end of the peak represents a smaller overall portion of demand, meaning there is less relative opportunity for deferring service until demand tails off, leading to a higher proportion of lost business from high-value parties. Thus, we expect that:

Hypothesis 6: Cherry-picking will be viable at lower levels of excess demand with longer peaks.

Ramp-up duration refers to the length of time from the restaurant's opening time on a day until the customer arrival rate reaches its peak. We assume that the customer arrival rate builds at a linear rate over the ramp-up period. With a slower build-up rate, it takes longer for the restaurant to reach full capacity, and is less likely that higher-value parties would be lost even if the lower-value parties are served. We expect, then, that:

Hypothesis 7: Cherry-picking will be viable at lower levels of excess demand with shorter rampup periods.

Table size proportions refer to the space required per seat, across the different table sizes that can be used in a restaurant. This factor had two levels: proportional and non-proportional; the latter where the space required per seat declined with larger tables. Table 3 reports the values we used for the factor's levels. When the table size is proportional to the number of seats, we have a conservation of seating capacity across alternative configurations. For example, a table of 10 seats could be replaced by five 2-seats tables; by one 4-seat and three 2-seat tables; by two 4-seat and one 2-seat tables; by one 4-seat and one 6-seat table; by one 6-seat and two 2-seat tables; or by one 8-seat and one 2-seat table. All of these alternatives 
maintain the 10 seat capacity of the original table. In contrast, when table size is non-proportional, then capacity is not preserved. Using the space requirements reported in Table 3, a 10-seat table could be replaced by three 2-seat tables; by one 4-seat and one 2-seat table; by two 4-seat tables; by one 6-seat and one 2-seat tables; or by one 8-seat table. These alternatives respectively have $6,6,8,8$, and 8 seats. With non-proportional space requirements, then, the loss of total seating capacity that occurs with the removal of a 10-seat table would make it harder to serve enough additional customers in the replacement tables to offset the lost business. Because of this, we expect that:

Hypothesis 8: Cherry-picking will be viable at lower levels of excess demand with proportional table sizes compared to non-proportional table sizes.

Simulation assumptions. We made 15 assumptions in the simulation study, none of which should be considered as nonstandard or as over limiting the applicability of our study. We assumed:

1. There were only parties of 1 to 10 people.

2. Parties would not be split across tables.

3. Tables had only even numbers of seats. This is largely equivalent to the restaurant using square or rectangular tables, rather than round or oval tables.

4. Tables could not be combined. Thompson (2002), in study of table combinability, found that restaurants with more than 50 seats (a cutoff that is smaller than the restaurants we simulate) generally are better off with a mix of table sizes, rather than having only small tables that can be combined together to seat larger parties. The reason for this is that productive time is lost when a table that frees up is placed “on hold,'” waiting for an adjacent table to become available so that the tables can be combined.

5. All demand was from walk-ins. The reason for this assumption is that taking reservations allows a restaurant much more control over how to best fill the restaurant (i.e., the basis for revenue management) and we wished to examine the phenomenon of cherry-picking in a more dynamic environment. 
6. Party arrivals followed a Poisson distribution, which is commonly observed service settings. The instantaneous mean arrival rate was a function of restaurant size, demand intensity, and the length of the ramp-up, peak and ramp-down periods.

7. Dining durations were lognormally distributed, reflecting the fact that some parties tend to linger over their meals, with mean values based on party size, as described earlier.

8. Random variation in dining duration for individual parties, based on a normal distribution with a 0.30 coefficient of variation, where the mean was equal to that for the size of the particular party (as described earlier). This value falls within the range of 0.16-0.50 reported in the literature (Bell and Pliner 2003; Kimes and Robson 2004).

9. An 1-hour ramp-down following the peak demand period, where the party arrival rate decreased linearly. We include the ramp-down period so that the restaurant has an opportunity to clear the waiting parties and note that any restaurant fortunate enough to have a demand level that results in waiting parties at closing time should consider extending its operating hours.

10. Random variation in individual parties’ willingness to wait for a table, based on a normal distribution with a 0.20 coefficient of variation, where the mean was set based on the party’s size (as described earlier).

11. Parties would wait up to their individual tolerance (identified in assumption \#10) and then depart if they had not been seated.

12. No limit on the number of parties that could be waiting. Restaurants commonly have areas, such as bars or waiting areas for customers, which can accommodate a limited number of waiting parties. Nonetheless, we made this assumption to avoid confounding effects, enabling us to focus only on the effects of differences in the waiting tolerances across parties.

13. Random variation in the check amount for individual parties, based on a normal distribution with a 0.25 coefficient of variation, where the mean was equal to that for the size of the party (as described earlier). While we are aware of no empirical studies on this parameter, the value we 
used with within the range of coefficients of variation of 0.15 and 0.30 used by Thompson and Sohn (2009).

14. Tight “right-sizing”' (Kimes 2004), which allows a party to be assigned to a table no bigger than the smallest table size in which the party fits (giving at most one empty seat in a table in our experiment, since we used only tables with even numbers of seats). Of the published studies, most are unclear as to any restrictions on party-sized-based table size restrictions. Field, McKnew, and Kiessler (1997), however, used restrictions similar to ours, where parties of four or fewer were seated in 4-tops, while parties of five and six were seated in 6-tops (they used only 4- and 6 tops). Thompson (2011) recently has shown that tight right-sizing yields revenue advantages over allowing parties to sit in tables larger than the minimum size that will accommodate the party.

15. A table assignment rule of assigning an available table to the largest waiting party that fits in the table, regardless of wait. Previous research frequently has used this rule (Kimes and Thompson 2004; Thompson 2002), with one exception (Field, McKnew, and Kiessler 1997), which used a rule based on selecting the party with the longest wait. Thompson (2011) has shown that the rule we employ here yields higher revenue than the longest wait rule.

Results from the simulation experiment. Table 4 presents the average lower bound on the percentage excess demand necessary to justify cherry-picking. Across all 384 scenarios, excess demand would have to be $128.0 \%$ of full demand to justify cherry-picking. While the largest differences between factor levels occurred for table space proportionality, all of the differences are consistent with our eight hypotheses:

We ran a multiple regression analysis, with percentage excess demand required to justify cherrypicking as the dependent variable, and the experimental factors being the independent variables (with the values shown in Table 5). The regression had an adjusted $\mathrm{R}^{2}$ of .752. Table 5 reports the parameter coefficients from the regression. All of the coefficients are significant at the .001 level, with the exception 
of the coefficient for mean party size and ramp-up duration, which were not significant at the .10 level. The signs of all the coefficients are consistent with our eight hypotheses.

Across the 384 scenarios, there were 14 instances when a lower bound on the excess demand to justify cherry-picking was at its overall lowest levels of either 0\% (2 instances) or25\% (12 instances). These instances were clustered: 7 of the 8 scenarios with the smaller restaurant size, smaller mean party size, larger differences in the average check across party sizes, shortest propensity to wait, and proportional table sizes had a lower bound of $25 \%$ excess demand. Interestingly, Casual Restaurant is similar to this cluster: it is smaller than the smaller restaurant size, its mean party size is 2.55 people, its average check difference exceeds that of the higher level, and its table sizes are roughly proportional. Moreover, it is located in a "restaurant strip" where potential customers have a range of choices at hand, and consequently are probably unwilling to wait more than about 30-40 minutes for a table.

Across the 384 scenarios, there were 117 instances with a lower bound on the excess demand to justify cherry-picking equal to the highest level we examined, at 200\% higher than full demand. These instances also were clustered: 77 occurred with the larger restaurant; 112 with non-proportional table sizes; 70 with lower differences in average checks across party sizes; 86 with smaller differences in duration across party sizes; and 73 with shorter duration of peak demand.

Accuracy of the cherry-picking viability-prediction heuristics. Table 6 reports the number of instances, across the 384 simulated scenarios, where the cherry-picking viability prediction heuristics yielded predictions that were below, within, or above the true limits on the excess demand that would justify cherry-picking. DmdHeur1 was optimistic, predicting that cherry-picking would be viable at lower levels of excess demand than actually was the case. DmdHeur2 fared much better: its predicted level of excess demand necessary to justify cherry-picking fell within the actual bounds about $42 \%$ of the time, with the remainder of the cases being split between predicting too high and predicting too low. We attribute the difference in accuracy between the two heuristics to DmdHeur2's ability to consider how 
customers’ willingness to wait for service reduces the amount of business lost when all tables are retained.

\section{Discussion}

Our international survey of restaurants indicated that the phenomenon of party-size-based cherrypicking occurs with some regularity, despite the phenomenon not being addressed previously in the literature. Even restaurants that do not actively discourage large parties from dining may indirectly accomplish that outcome, since the majority of respondents to the survey prefer to overestimate the waiting time for large parties.

To reiterate the findings from our simulation study, party size- based cherry-picking is viable at lower levels of excess demand with: smaller restaurants; smaller mean party sizes; greater differences in per-person average checks across party sizes; greater differences in dining duration across party sizes; lower customer tolerances for waiting; longer peak demand windows; short ramp-up periods; and tables with space requirements proportional to their number of seats. All of these factors were statistically significant except for mean party size and ramp-up duration. A reasonable question to ask, then, is: Does party-size-based cherry-picking make good business sense? The answer is: It depends. Certainly there are environments where cherry-picking is viable with relatively low levels of excess demand. For example, if a restaurant had the following characteristics it should consider cherry-picking customers based on party size: small size, small mean party size, large differences in the average per-person check across party sizes, short willingness of customers to wait, and proportional table sizes. Party-size-based cherry-picking is generally impractical when the average check per person and dining duration are both more similar across party sizes. Interestingly, there is good congruence between the results of the survey and the simulation results around the effect of restaurant size. The survey (Figure 2) showed that wait-time quotes consistent with cherry-picking occur more often in smaller restaurants than in larger restaurants. 
To use either of the cherry-picking viability prediction heuristics, or a more sophisticated simulation-based approach, a restaurant would need to have some means of tracking excess demand. This excess demand represents the true, unconstrained level of demand, in contrast to the constrained demand information that appears, for example, in the point-of-sale system. Estimating excess demand would require tracking the number of people who attempt to enter the restaurant but, when quoted an estimated wait time, decline to put their name on a wait list. In addition, it would be important to track the number of people who put their names on the wait list but leave before being seated. However, even with both types of information the estimate of true demand is likely to be low, because of people who decide not to approach a host or hostess if the restaurant appears excessively busy.

There are at least nine extensions suggested by our work. First, some restaurants will only serve large parties during off peak periods, which can be considered to be cherry-picking based on time and party size. Time-and-party-size-based cherry-picking offers a transition between no cherry-picking and full (party-size-based) cherry-picking. It would also offer restaurateurs an opportunity to tell large parties that there are times that they would be served, potentially mitigating issues with customer dissatisfaction. This could be particularly relevant, since cherry-picking is likely to be much more obvious to customers in a restaurant—with potential negative implications — because the status of a restaurant is visible, as compared to cherry-picking in hotels, where customers cannot see the status of a virtual queue of reservation requests. It would be interesting to see, then, how to best apply such timing restrictions. Second, one could attempt to make the cherry-picking decision dependent on the current state of the restaurant, specifically with respect to which tables are occupied and which are unoccupied. Analytical models may prove useful in that regard. A worthwhile goal would be developing decision rules to aid managers, perhaps stated in the form "'serve a large party if you have x unoccupied tables." Third, it would seem likely that customers' willingness to wait is not static across time as we have assumed. For example, we expect that customers would tolerate a longer wait early in an evening dining period than they would tolerate later in the evening. Dealing with time-dependent waiting tolerances is 
straightforward in a simulation. While the second heuristic we evaluated for predicting the level of excess demand necessary for cherry-picking could easily be adapted for this case, it remains to be seen how it would perform. Fourth, we assumed that waiting tolerance grew linearly with party size increase. In reality, this may not be a linear function and so it would be useful to explore the effects on cherry-picking of other functional relationships. Fifth, we considered only the case where one table size was being eliminated. It would be interesting to examine more extensive cherry-picking, where two or more table sizes were being considered for elimination. It seems obvious that the level of excess demand would have to be even larger to justify more extensive cherry-picking. Sixth, while we assumed linear relationships between party size and dining duration and the average check per person, the data available from the few studies that have reported this information (Kimes and Robson 2004; Kimes and Thompson 2004) seems to be nonlinear. A nonlinear relationship would clearly affect the level of excess demand necessary to justify cherry-picking. Seventh, given the computational effort involved in identifying the level of excess demand necessary to justify cherry-picking, the development and testing of more viability-prediction heuristics might be worthwhile, to assist restaurateurs in determining whether party-size based cherrypicking is indeed viable for their restaurants. Eighth, some restaurants implement cherry picking for parties that best fit a table (e.g., picking parties of two and four over parties of one and three). While such cherry-picking may be effective in restaurants using reservations, since the customers cannot see who is waiting, it may be less practical for restaurants dependent on walk-in customers; nonetheless, it would be interesting to examine such contexts. Ninth, while our focus here has been on the level of excess demand that is necessary to justify cherry-picking, there are other important considerations. Primarily, these relate to the potential downside the restaurant may experience when customers are aware that cherry-picking is being implemented. Thus, it would be useful to examine the customer-management strategies used by restaurants that implement cherry-picking. 


\section{Conclusion}

In this paper, we have examined the issue of cherry-picking customers based on party size- the actions of serving only the most valuable customers and denying service to less valuable customers—in the contexts of restaurants. While these actions are at the core of revenue management, we are, to our knowledge, the first to examine the phenomenon in restaurants. Two common characteristics of customers in restaurants set the stage for party-size-based cherry-picking in restaurants: larger parties take longer to dine and spend less per person than smaller parties. The findings of a survey of restaurants from around the world exhibited characteristics of cherry-picking. Our focus has been on identifying the level of excess demand that is necessary to justify turning away the lower-value customers. Using a simulationbased study of 384 restaurant environments, we found that cherry-picking is more viable in certain environments: larger variation in per-person checks across party sizes, larger variation in dining durations across party sizes, smaller restaurants, less willingness on the part of customers to wait for tables, longer peak-demand windows, and with tables that have space requirements proportional to their number of seats. We evaluated two heuristics for predicting the level of excess demand that is necessary to justify cherry-picking. The heuristic that incorporated information on customers' willingness to wait for a table proved to be the far more accurate. Our hope is that this study will stimulate follow-up work, since there are a number of avenues for future research that appear promising. 


\section{References}

Anonymous (2010), 2010 Restaurant Industry Pocket Factbook. Washington DC: National Restaurant Association.

Bell, Rick and Patricia L. Pliner (2003), “'Time to Eat: The Relationship Between the Number of People Eating and Meal Duration in Three Lunch Settings,’’ Appetite, 41, 215-218.

Bertsimas, Dimitras and Romy Shioda (2003), “'Restaurant Revenue Management,’’ Operations Research, 51 (3), 472-486.

Field, Arthur, Mark McKnew, and Peter Kiessler (1997), “'A Simulation Comparison of Buffet Restaurants: Applying Monte Carlo Modeling,'” Cornell Hotel and Restaurant Administration Quarterly, 38 (6), 68-79.

Kelly, Thomas J., Nicholas M. Kiefer, and Kenneth Burdett (1994), “A Demand-Based Approach To Menu Pricing,’' Cornell Hotel and Restaurant Administration Quarterly, 35 (1), 48-52.

Kimes, Sheryl E. (2004), “'Restaurant Revenue Management,’’ A Research Report of The Center for Hospitality Research, Cornell University, 4 (2).

— Expected Meal Duration for Restaurant Revenue Management,’’ Journal of Revenue and Pricing Management, 1 (3), 220-233.

and Stephani K. A. Robson (2004), “The Impact of Restaurant Table Characteristics on Meal Duration and Spending,' Cornell Hotel and Restaurant Administration Quarterly, 45 (4), 333346.

- and Gary M. Thompson (2004), “Restaurant Revenue Management at Chevys: Determining the Best Table Mix,’ Decision Sciences, 35 (3), 371-391.

, Richard B. Chase, Sunmee Choi, Philip Y. Lee, and Elizabeth N. Ngonzi (1998), “'Restaurant Revenue Management: Applying Yield Management To The Restaurant Industry,’’ Cornell Hotel and Restaurant Administration Quarterly, 39 (3), 32-39. 
Kumar, V., Katherine N. Lemon, and A. Parasuraman (2006), “Managing Customers for Value: An Overview and Research Agenda,’' Journal of Service Research, 9 (November), 87-94.

McGuire, Kelly and Sheryl E. Kimes (2006), “The Perceived Fairness of Waitlist Management Techniques for Restaurants,'” Cornell Hotel and Restaurant Administration Quarterly, 45 (2), 121-134.

Ostrom, Amy L., Mary Jo Bitner, Stephen W. Brown, Kevin A. Burkhard, Michael Goul, Vicki SmithDaniels, Haluk Demirkan, and Elliot Rabinovich (2010), “'Moving Forward and Making a Difference: Research Priorities for the Science of Service,’ Journal of Service Research, 13 (February), 4-36.

Robson, Stephani K. A. and Sheryl E. Kimes (2009), “Don’t Sit so Close to Me: Restaurant Table Characteristics and Guest Satisfaction,’ A Research Report of The Center for Hospitality Research, Cornell University, 9 (2).

Smith, Barry C., John F. Leimkuhler, and Ross M. Darrow (1992), “'Yield Management at American Airlines,'’ Interfaces, 22 (1), 8-31.

Thompson, Gary M. (2011), “'Inaccuracy of the “'Naive Table Mix”' Calculations,” Cornell Hotel and Restaurant Administration Quarterly. (2002), “'Optimizing a Restaurant’s Seating Capacity: Use Dedicated or Combinable Tables?’’ Cornell Hotel and Restaurant Administration Quarterly, 43 (4), 48-57.

—_ and Heeju J. Sohn (2009), “Time- and Capacity-Based Measurement of Restaurant Revenue,” Cornell Hospitality Quarterly, 50 (4), 520-539.

— and Robert J. Kwortnik, Jr. (2008), “Pooling Restaurant Reservations to Increase Service Efficiency,'’ Journal of Service Research, 10 (4), 335-346. 


\section{Appendix}

\section{Algorithm for the Waiting Tolerance based Cherry-Picking Viability Prediction Heuristic}

Before describing the steps in the heuristic, we shall first define some terms the heuristic uses:

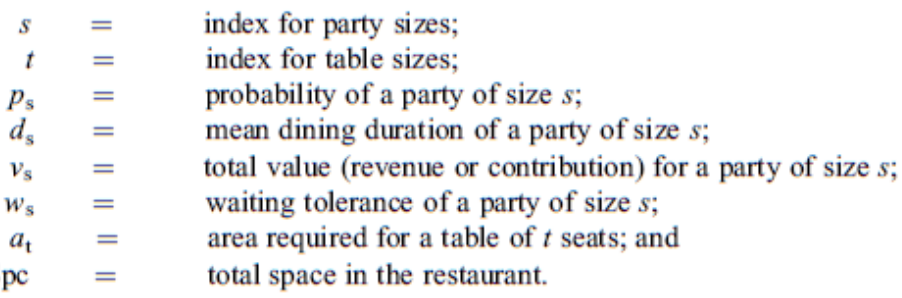

The steps in DmdHeur2 are

1. Initialize RampUpDur, PeakDur, RampDnDur and InitMaxPartyArvRate.

2. For each table size $t$, set the average wait tolerances ( TblWaitTol $_{t}$ ), average values

$\left(\right.$ TblVal $\left._{t}\right)$, average durations $\left(\right.$ TblDur $\left._{t}\right)$, and expected space $\left(\right.$ TblSpace $\left._{t}\right)$ as:

$$
\begin{aligned}
& \text { TblWaitTol }_{t}=\left(p_{t-1}{ }^{*} w_{t-1}+p_{t}^{*} w_{t}\right) /\left(p_{t-1}+p_{t}\right) ; \\
& \text { TblVal }_{t}=\left(p_{t-1} v_{t-1}+p_{t}^{*} v_{t}\right) /\left(p_{t-1}+p_{t}\right) ; \\
& \text { TblDur }_{t}=\left(p_{t-1} * d_{t-1}+p_{t}^{*} d_{t}\right) /\left(p_{t-1}+p_{t}\right) ; \text { and } \\
& \text { TblSpace }_{t}=\left(p_{t-1}+p_{t}\right)^{*} a_{t} .
\end{aligned}
$$

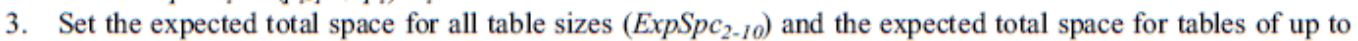
8 seats $\left(\operatorname{ExpSpc}_{2-8}\right)$ as:

$$
\begin{aligned}
& \text { ExpSpc } 2-10=\sum_{t=2,4,6,8,10} \text { TblSpace }_{t} \text { and } \\
& \text { ExpSpc }_{2-8}=\sum_{t=2,4,6,8} \text { TblSpace }_{t} .
\end{aligned}
$$

4. For each table size $t$, set the available capacity (in tables) when all tables are used (TblAvlCap2-10 $)$ and the available capacity (in tables) when only tables of 2-8 seats used (TblAvlCap 2-8 ${ }_{t}$ ) as:

TblAvlCap2-10 $=$ TtlSpc $*$ TblSpace $_{t} /\left(\right.$ ExpSpc $\left._{2-10} * a_{t}\right)$ and

TblAvlCap2- $8_{t}=$ TtlSpc $*$ TblSpace $_{t} /\left(\operatorname{ExpSpc}_{2-8} * a_{t}\right)$.

5. Initialize $d m$ dinc to 0 .

6. Set $d$ mdinc $=$ dmdinc +0.01 .

7. Initialize TotLost T-10 $_{10}$ to 0 and Tot Lost $_{l-8}$ to 0 .

8. Set MaxPartyArvRate $=$ InitMaxPartyArvRate $*(1+$ dmdinc $)$.

9. Loop for $t=2$ through 10 , incrementing by 2

9.a. Set Tot Lost1 $10_{t}=$ TotLost $1-8_{t}=0$.

9.b. Loop for $m=1$ to 1440

9.b.1. Set AvlCap1-10 $=$ TblAvlCap2-10 .

9.b.2. Set AvlCap1- $8_{m}=$ TblAvlCap2- ${ }_{t}$.

End loop [9.b.]

9.c. Loop for $m=1$ to RampUpDur + PeakDur + RampDnDur

9.c.1. If ( $m \leq$ RampUpDur) then

Set toAssign $=$ MaxPartyArvRate $*\left(p_{t-1}+p_{t}\right) * m /$ RampUpDur.

Else, if ( $m \leq$ RampUpDur + PeakDur $)$ then Set toAssign $=$ MaxPartyArvRate ${ }^{*}\left(p_{t-1}+p_{t}\right)$.

Else

Set toAssign $=$ MaxPartyArvRate $*\left(p_{t-1}+p_{t}\right) *($ RampUpDur + PeakDur + RampDnDur $+1-m) /$

End if RampDnDur.

9.c.2. Initialize toAssign 1-10 equal to toAssign.

9.c.3. Loop for $i=m$ to $m+\left\lfloor\right.$ TblDur $_{t}+$ TblWaitTol $\left._{t}+0.5\right\rfloor-1$

9.c.3.a. If $($ AvlCap 1-10 $>0)$ then

9.c.3.a.1. Set Assigned $=\min ($ AvlCap1-10, toAssign 1-10)

9.c.3.a.2. Loop for $k=i$ to $i+\left\lfloor T b l D u r_{t}+0.5\right\rfloor-1$ 


\section{Appendix con't}

9.c.3.a.2.a. Set AvlCap1-10 $=$ AvlCap1-10 - Assigned. End loop (9.c.3.a.2)

9.c.3.a.3. Set toAssign1-10 = toAssign1-10 - Assigned . End if (9.c.3.a)

End loop (9.c.3)

9.c.4. Set TotLost1-10 $=$ TotLost1-10 + Assign.

9.c.5. If $\mathrm{t}=10$, skip steps 9.c.6 through 9.c.8.

9.c.6. Initialize toAssign 1-8 equal to toAssign.

9.c.7. Loop for $i=m$ to $m+\left\lfloor\right.$ TblDur $_{t}+$ TblWaitTol $\left._{t}+0.5\right\rfloor-1$

9.c.7.a. If $\left(\right.$ AvlCap 1- $\left.\delta_{i}>0\right)$ then

9.c.7.a.1. Set Assigned $=\min \left(\right.$ AvlCap1- $8_{i}$, toAssign 1-8).

9.c.7.a.2. Loop for $k=i$ to $i+\left\lfloor\right.$ TblDur $\left._{t}+0.5\right\rfloor-1$

9.c.7.a.2.a. Set AvlCap1- $\delta_{i}=$ AvlCap1- $\delta_{i}$-Assigned.

End loop (9.c.7.a.2)

9.c.7.a.3. Set toAssign1-8= toAssign 1-8 - Assigned. End if (9.c.7.a)

End loop (9.c.7)

9.c.8. Set TotLost $1-8_{t}=$ TotLost $1-8_{t}+$ Assign .

End loop (9.c.)

9.d. Set TotLost1-10 = TotLost1-10 + TotLost 1-10 ${ }_{t}^{*}$ TblVal $_{t}$.

9.e. If $(\mathrm{t}<10)$ then Set TotLost $1-8=$ TotLost $1-8+$ TotLost $1-8{ }_{t}^{*}$ TblVal $_{t}$.

End loop (9)

10. If $(($ TotLost $1-10>$ TotLost $1-8)$ OR $($ dmdinc $>5))$ then

Else

Return dmdinc. Stop.

Return to Step 6.

End if 
Table 1. Geographical Distribution of Respondents.

\begin{tabular}{lcc}
\hline Country/Region & $\begin{array}{c}\text { Percentage of } \\
\text { Respondents }\end{array}$ & $\begin{array}{c}\text { Average No. } \\
\text { Seats }\end{array}$ \\
\hline Asia & 24.4 & 245 \\
U.S.A. & 22.0 & 181 \\
\hline Europe & 21.5 & 175 \\
\hline Canada & 6.8 & 197 \\
\hline Australia \& Pacific Islands & 5.9 & 309 \\
\hline South America & 5.9 & 205 \\
\hline Mideast & 5.4 & 299 \\
\hline Caribbean Islands & 2.9 & 166 \\
\hline Mexico & 2.9 & 184 \\
Africa & 2.0 & 139 \\
\hline Central America & 0.5 & 129 \\
\hline
\end{tabular}

Table 2. Experimental Factors in the Simulation Experiment

\begin{tabular}{|c|c|}
\hline Experimental Factor & \# Levels: Levels \\
\hline Restaurant size & $\begin{array}{l}\text { 2: Approximately } 30 \text { and } 60 \text { parties seated } \\
\text { simultaneously }\end{array}$ \\
\hline Mean party size & 2: 2.5 and 3.0 people \\
\hline $\begin{array}{l}\text { Average check variation } \\
\text { across party sizes }\end{array}$ & $\begin{array}{l}\text { 2: } \$ 10 \text { average spend per person for par- } \\
\text { ties of one, decreasing linearly to } \$ 9 \\
\text { and } \$ 8 \text { average spend per person for } \\
\text { parties of } 10\end{array}$ \\
\hline Propensity to wait & $\begin{array}{l}\text { 3: Long, medium, and short: wait toler- } \\
\text { ances of } 105-120,45-60 \text { minutes, and } \\
\text { 15-30 minutes, respectively, for parties } \\
\text { of } 1-10 \text { (increasing linearly by party } \\
\text { size) }\end{array}$ \\
\hline $\begin{array}{l}\text { Duration variation across } \\
\text { party sizes }\end{array}$ & $\begin{array}{l}\text { 2: Ratio of duration for a party of } 10 \\
\text { compared to the duration for a party of } \\
\text { I equal to } 1.5 \text { and } 2.0\end{array}$ \\
\hline Peak demand length & $2: 2$ and 4 hours \\
\hline Ramp-up duration & 2: 1 and 2 hours \\
\hline Table space proportions & $\begin{array}{l}\text { 2: Space requirements proportion and } \\
\text { nomproportional to the number of } \\
\text { seats }\end{array}$ \\
\hline
\end{tabular}


Table 3. Parameter Levels for Probabilities of Party Sizes and Table Space Requirements

\begin{tabular}{|c|c|c|c|c|}
\hline \multirow{2}{*}{$\begin{array}{l}\text { Party Size } \\
\text { or Table Seats }\end{array}$} & \multicolumn{2}{|c|}{$\begin{array}{c}\text { Party Size } \\
\text { Probabilities, by } \\
\text { Mean Party Size }\end{array}$} & \multicolumn{2}{|c|}{$\begin{array}{c}\text { Table Space } \\
\text { Requirements }\left(\mathrm{ft}^{2}\right)\end{array}$} \\
\hline & 2.5 & 3.0 & Proportional & Nonproportional \\
\hline I & 0.31 & 0.25 & & \\
\hline 2 & 0.42 & 0.29 & 30 & 45 \\
\hline 3 & 0.10 & 0.17 & & \\
\hline 4 & 0.05 & 0.12 & 60 & 70 \\
\hline 5 & 0.03 & 0.05 & & \\
\hline 6 & 0.02 & 0.03 & 90 & 95 \\
\hline 7 & 0.02 & 0.025 & & \\
\hline 8 & 0.02 & 0.025 & 120 & 120 \\
\hline 9 & 0.02 & 0.025 & & \\
\hline 10 & 0.01 & 0.015 & 180 & 145 \\
\hline
\end{tabular}

Table 4. Average Lower Bound on the Percentage Excess Demand Necessary to Justify CherryPicking, by Level of the Experimental Factors

\begin{tabular}{|c|c|c|}
\hline Factor & Level & $\begin{array}{l}\text { Average Percentage } \\
\text { Excess Demand } \\
\text { Necessary to Justify } \\
\text { Cherry-Picking }\end{array}$ \\
\hline $\begin{array}{l}\text { Restaurant size } \\
\text { (approximate table } \\
\text { count) }\end{array}$ & $\begin{array}{l}30 \\
60\end{array}$ & $\begin{array}{l}113.9 \\
142.1\end{array}$ \\
\hline Mean party size (people) & $\begin{array}{l}2.5 \\
3.0\end{array}$ & $\begin{array}{l}123.7 \\
132.3\end{array}$ \\
\hline $\begin{array}{l}\text { Average check variation } \\
\text { across party sizes }\end{array}$ & $\begin{array}{l}\text { Smaller } \\
\text { Larger }\end{array}$ & $\begin{array}{l}138.0 \\
118.0\end{array}$ \\
\hline \multirow[t]{2}{*}{ Propensity to wait } & $\begin{array}{l}\text { Long } \\
\text { Medium }\end{array}$ & $\begin{array}{l}146.1 \\
124.4\end{array}$ \\
\hline & Short & 113.5 \\
\hline $\begin{array}{l}\text { Duration variation across } \\
\text { party sizes }\end{array}$ & $\begin{array}{l}\text { Smaller } \\
\text { Larger }\end{array}$ & $\begin{array}{l}145.3 \\
110.7\end{array}$ \\
\hline Peak demand length (hours) & $\begin{array}{l}2 \\
4\end{array}$ & $\begin{array}{l}145.1 \\
110.9\end{array}$ \\
\hline Ramp-up duration (hours) & $\begin{array}{l}1 \\
2\end{array}$ & $\begin{array}{l}125.7 \\
130.3\end{array}$ \\
\hline Table space proportions & $\begin{array}{l}\text { Proportional } \\
\text { Nonproportional }\end{array}$ & $\begin{array}{r}89.1 \\
166.9\end{array}$ \\
\hline
\end{tabular}

Note. ${ }^{2}$ Average lower bound percentage of full demand. 
Table 5. Regression Average Percentage Increase in Demand Necessary to Justify Cherry-Picking, by Level of the Experimental Factors

\begin{tabular}{|c|c|}
\hline Experimental Factor & Regression Coefficient \\
\hline Intercept & $214.44^{* 0 * *}$ \\
\hline $\begin{array}{l}\text { Restaurant size (measured as the } \\
\text { number of parties that could be } \\
\text { seated simultaneously; i.e., } 30 \text { and } 60 \text { ) }\end{array}$ & $0.9375^{* 016 k}$ \\
\hline Mean Party Size (in people; i.e., 2.5 and 3.0) & 3.0849 \\
\hline $\begin{array}{l}\text { Average check variation across party sizes } \\
\text { (measured as the difference in average } \\
\text { check value per person, for parties } \\
\text { differing in size by one person; i.e., } \\
\$ 0.111 \text { and } \$ 0.222 \text { ) }\end{array}$ & $-180.65^{* * * *}$ \\
\hline $\begin{array}{l}\text { Propensity to wait (in minutes, for parties } \\
\text { of one; i.e., 105, } 45 \text {, and 15) }\end{array}$ & $0.35256^{* * 0 *}$ \\
\hline $\begin{array}{l}\text { Duration variation across party sizes } \\
\text { (measured as the ratio of duration for a } \\
\text { party of } 10 \text { compared to the duration for } \\
\text { a party of I; i.e., } 1.5 \text { and } 2.0 \text { ) }\end{array}$ & $-69.271^{* 010 \%}$ \\
\hline Peak demand length (in hours; i.e., 2 and 4) & $-17.057^{* 010 *}$ \\
\hline Ramp-up duration (in hours; i.e., I and 2) & 4.6875 \\
\hline $\begin{array}{l}\text { Table space proportions (proportional }=0 ; \\
\text { nonproportional }=\mathrm{I} \text { ) }\end{array}$ & $77.865^{\text {*owk }}$ \\
\hline
\end{tabular}

Note. ${ }^{* 10 k p} p<.001$

Table 6. Accuracy of the Excess Demand Prediction Heuristics

\begin{tabular}{lcr}
\hline Outcome & DmdHeur I & DmdHeur2 \\
\hline Prediction below range* & $382(99.5 \%)$ & $129(33.6 \%)$ \\
Prediction within range & $2(0.5 \%)$ & $160(41.7 \%)$ \\
Prediction above range & $0(0.0 \%)$ & $95(24.7 \%)$ \\
\hline
\end{tabular}

Note. ${ }^{2}$ Of the 384 scenarios in the simulation experiment. 


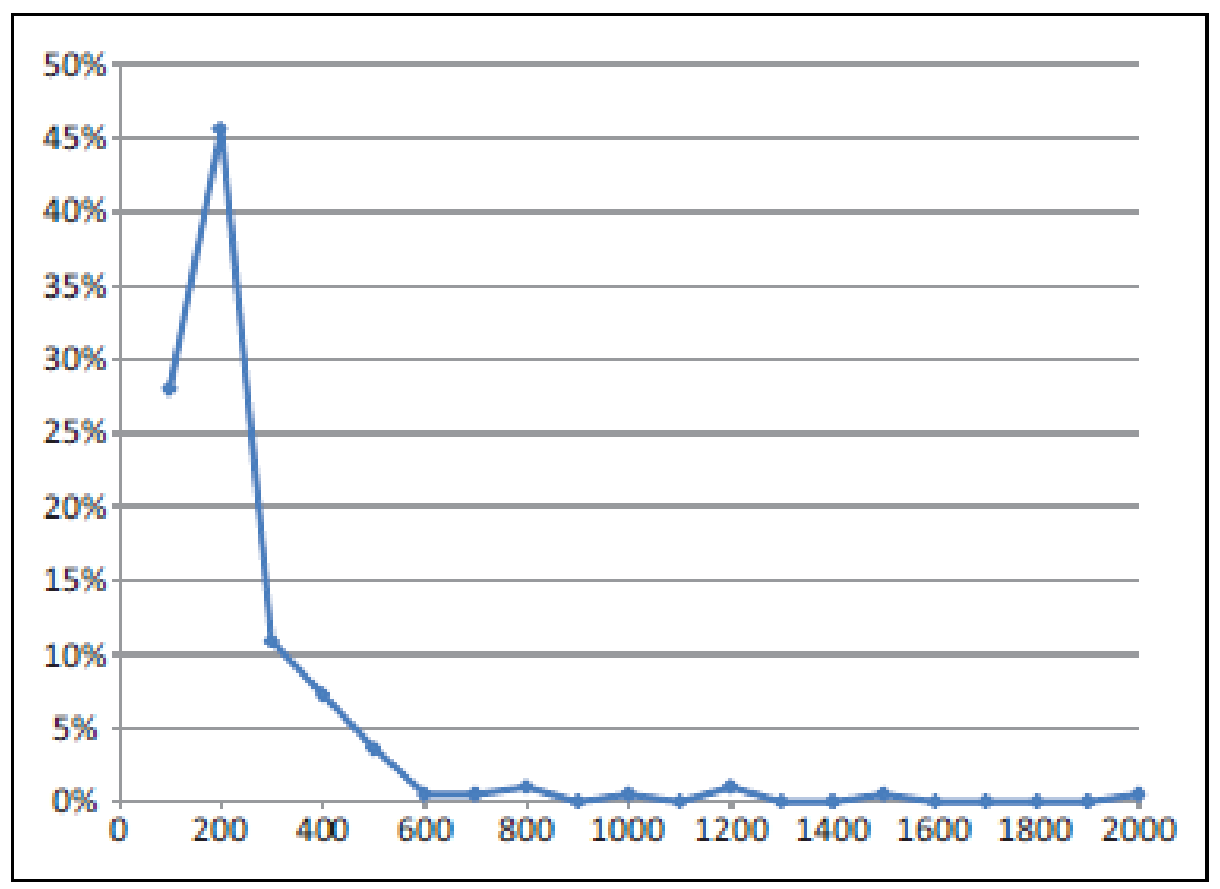

Figure 1. A relative frequency histogram of the number of restaurant seats. 


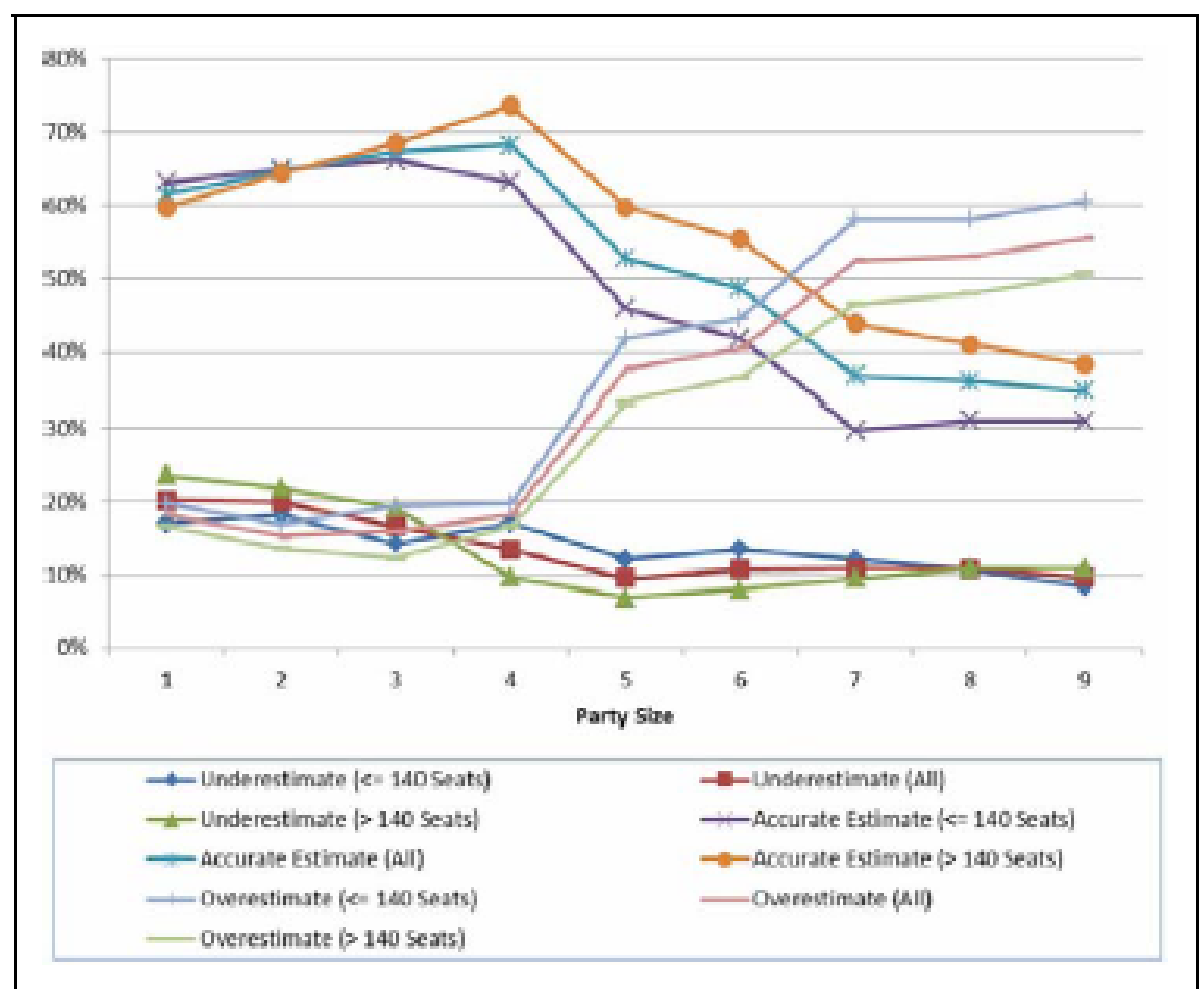

Figure 2. Preferred wait time quote estimates, by party size and restaurant size. 


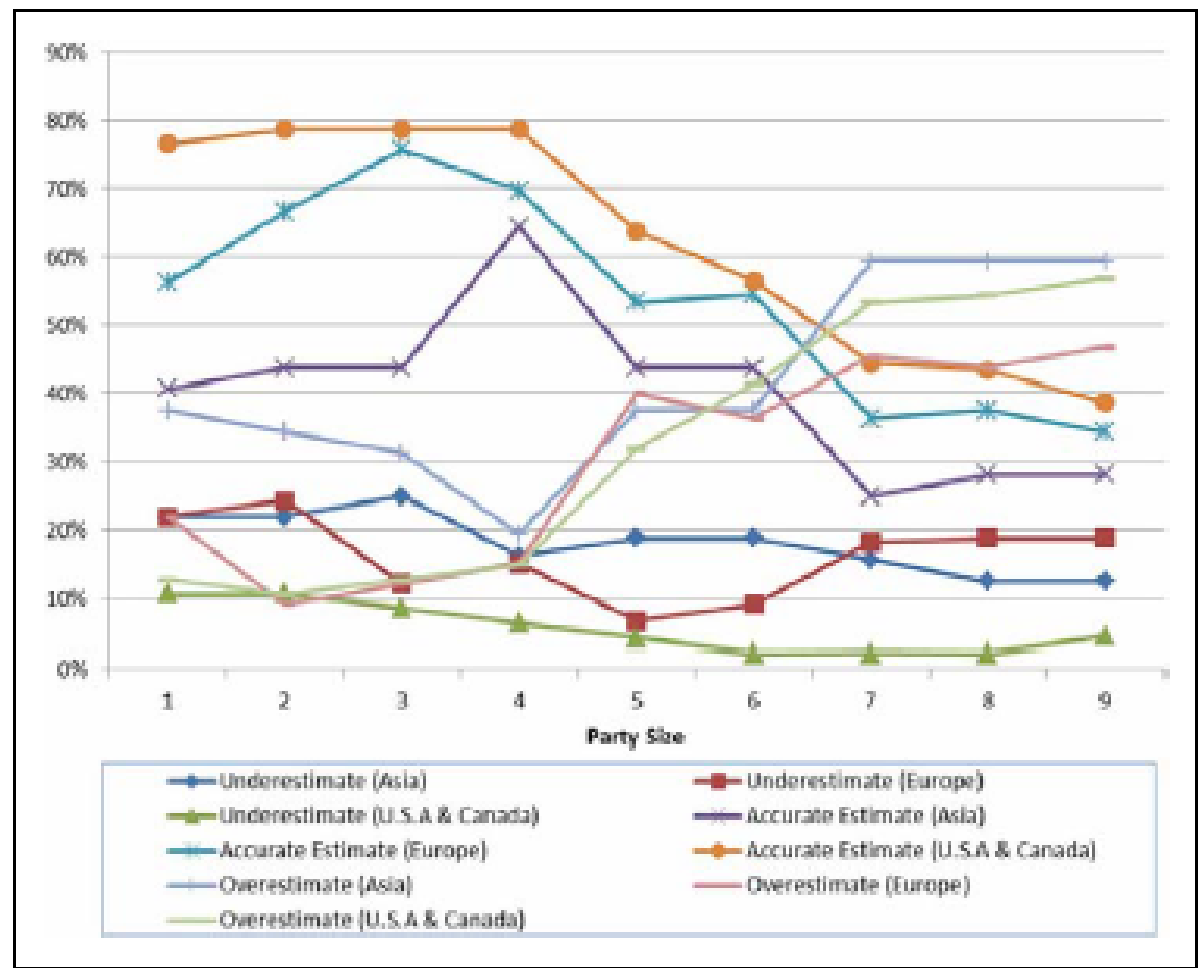

Figure 3. Preferred wait time quote estimates, by party size and geographical location. 